\title{
Lung Lobar Slippage Assessed with the Aid of Image Registration
}

\author{
Youbing Yin $^{1}$, Eric A. Hoffman ${ }^{2, \star}$, and Ching-Long Lin $^{1}$ \\ ${ }^{1}$ Department of Mechanical \& Industrial Engineering \\ ${ }^{2}$ Departments of Biomedical Engineering, Radiology, and Medicine \\ The University of Iowa, Iowa City, IA 52242 \\ \{youbing-yin, eric-hoffman, ching-long-lin\}@uiowa.edu
}

\begin{abstract}
We present a registration algorithm that can handle the discontinuity of deformation with an ultimate goal to investigate how pulmonary lobes deform to accommodate chest wall shape changes. We first show that discontinuities can exist in both normal and tangent directions. Such discontinuities are accounted for by a spatially varying diffusive regularization which restricts smoothing inside objects. Meanwhile, a distance term is combined with the sum of squared intensity differences (SSD) to explicitly match corresponding interfaces and intensity patterns. The capability of this new method is demonstrated using two-dimensional (2-D) synthetic examples with complete or incomplete "fissures" and threedimensional (3-D) computed tomography (CT) lung datasets.
\end{abstract}

\section{Introduction}

Nonrigid image registration has become an important non-invasive tool to assess organ motion from a pair of medical images. Most earlier registration algorithms assume or imply a continuous and smooth deformation field. However, those algorithms might introduce unphysiological artifacts near object interfaces if the adjacent objects slip against each other. Thus, developing physiologically meaningful registration algorithms that account for discontinuities is becoming an important issue 123456 . Of particular interest to this paper is discontinuity near lobar fissures in human lungs.

It is well known that human lungs are separated into five lobes by means of infolded reflections of the visceral pleura and lobes can slide against the chest wall and adjacent lobes [7. Such motion may provide a means to reduce lung parenchymal distortion and avoid regions of high local stress. Conversely, it has previously been shown that lobar fissures fibrose and essentially disappear in animals (sloths) which undergo very little chest wall shape changes [8]. Recently, some registration algorithms have been proposed to account for discontinuity of deformation near lung borders or lobar fissures [2 3/4 5]. In [2 34], discontinuities are accounted for by registering regions of interest alone or separately with segmentation masks. Voxels outside of the region of interest are not taken into

^ E.A. Hoffman is shareholder in VIDA Diagnostics, Inc.

T. Jiang et al. (Eds.): MICCAI 2010, Part II, LNCS 6362, pp. 578-585, 2010.

(C) Springer-Verlag Berlin Heidelberg 2010 
consideration during registration or are set to a uniform value before registration to form a high intensity contrast on the border. By this means these methods avoid inter-object regularization and match corresponding borders with an implicit penalty of the high intensity contrast between object and "background". However, such mask-based registration methods require a complete interface. Commonly, incomplete lobar fissures are observed in human lungs and the degree of incompleteness ranges from nearly complete absence to nearly complete presence of the fissure [9. This means that lobes might be only partially separated and, thus, mask-based registration methods discussed above would be incapable of dealing with incomplete fissures. Different from mask-based registration, Kabus [1] introduced a spatially dependent weighting function to suppress the influence of smoothness near the interfaces of neighboring objects, thus, allowing for opening or closing of a gap between regions. Recently, Schmidt-Richberg et al. [5]6] proposed a direction-dependent regularization that restricts smoothing in normal directions while allowing discontinuity in tangent directions. An underlying assumption for their approach [5] is that normal components of displacements on the interface of neighboring objects are continuous. However, this assumption might not be true for lobar fissures since normal directions of fissures change during respiration, leading to discontinuities in both normal and tangent directions (it will be explained in details later). Furthermore, Schmidt-Richberg et al.'s approach cannot ensure corresponding interfaces to be matched between two images if the intensity contrast near the interface is weak, such as in the case for CT images of lobar fissures.

In this paper, we present a novel registration algorithm to deal with discontinuity of deformation occurring near lobar fissures. We show that discontinuities can exist in both normal and tangent directions and then present the new registration to account for such discontinuities. The proposed method is validated using two-dimensional (2-D) synthetic examples with complete or incomplete "fissures" and then applied to three-dimensional (3-D) computed tomography (CT) lung datasets.

\section{Methods}

\subsection{Background}

Given a pair of $N$-dimensional ( $N$-D) images $R$ and $F$, referred to as the reference and floating images, the goal of a registration algorithm is to find a deformation field to match images $R$ and $F$. If there is only one homogeneous object, we should expect a smooth and continuous deformation field. However, slippage or discontinuity may occur on interfaces if there are more than one object. Fig 1 illustrates three sliding cases, where the interfaces before and after motions are denoted by $\partial \Omega_{1}$ and $\partial \Omega_{2}$, respectively. A pair of adjacent points $p_{+}$and $p_{-}$on $\partial \Omega_{1}$ is deformed into corresponding points $q_{+}$and $q_{-}$on $\partial \Omega_{2}$. Due to slippage, $q_{+}$and $q_{-}$are separated but still locate on $\partial \Omega_{2}$. In left panel of Fig 1 interfaces before and after motion are planes with unchanged normal directions; in the middle both interfaces are planes but normal directions are different; and in the 

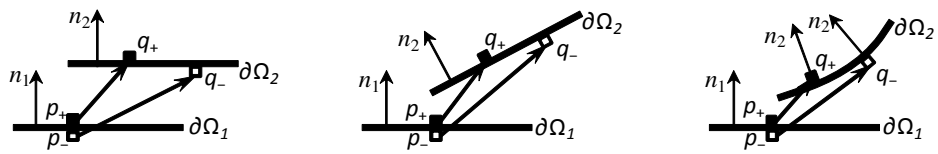

Fig. 1. A sketch illustrating sliding in three cases. Left: interfaces before and after motions are planes with unchanged normal directions; Middle: interfaces before and after motions are planes but with changed normal direction; Right: an initial plane interface is deformed into a curved surface after motion.

right an initial plane interface is deformed into a curved surface with different normal direction at $q_{+}$and $q_{-}$. It is clear that normal components (with respect to $n_{1}$ ) of displacements for $p+$ and $p$ - are continuous only for the first case while discontinuities exist in both normal and tangent directions for the other two cases where the normal directions change. Based on these observations, we should not expect continuous normal components of displacement near lobar fissures since their motions are usually complex with changed normal directions. Thus, a new registration method is needed to investigate discontinuity of deformation between lung lobes.

\section{$2.2 \quad$ Registration Method}

Image registration is a process of determining an optimal deformation field to match images $R$ and $F$. For non-parametric registration algorithms, deformation field is usually expressed in the form of displacement field $s: p \rightarrow s(p)$ for each voxel $p . s(\cdot)$ is usually solved by minimizing a global energy

$$
E=\operatorname{Sim}(R, F, s)+\frac{1}{\sigma_{T}^{2}} \operatorname{Reg}(s),
$$

where Sim is the similarity measure that defines how well images $R$ and $F$ match. $R e g$ is the regularization term that avoids unstable and non-smooth solutions and $\sigma_{T}$ is a weight coefficient to control the amount of regularization.

In order to capture discontinuities of deformation in both the normal and tangent directions, as we observe in Section 2.1. we need to suppress the regularization near the interface of neighboring objects while maintain regularization inside objects. Meanwhile, we have to ensure corresponding interfaces from images $R$ and $F$ to be matched to prevent gaps or intersections. For the first point, we adopt a spatially variable diffusive regularization:

$$
\operatorname{Reg}(s)=\sum_{l=1}^{N} \int_{\Omega} \beta(p)\left\|\nabla s_{l}(p)\right\|^{2},
$$

where $\beta(p)$ is a varying weight coefficient and $s_{l}$ is the $l$ th component of $s$. A desirable $\beta$ should restrict the regularization inside objects and one of choices is a Dirac-shaped function [15]: $\beta(p)=1 /\left(1+\alpha n \exp \left(-\alpha \phi_{R}^{2}(p)\right)\right.$, where $\alpha$ and $n$ 
are control parameters and we choose $\alpha=n=1000$ in this work. $\phi_{R}(p)$ is the Eucilidean distance at voxel $p$ to its closest point on the interface in image $R$.

For the similarity term in Eq. (1), we combine the sum of squared intensity differences (SSD) and a distance penalty term. The distance term measures distances of all points on an interface in image $R$ to the corresponding interface in image $F$ and the idea is similar to surface matching algorithms using distance transformation [10]. The combined similarity measure is in the form of

$$
\operatorname{Sim}(R, F, s)=\int_{\Omega}\|R-F \circ s(p)\|^{2}+\gamma \int_{\partial \Omega}\left\|\phi_{F} \circ s(p)\right\|^{2},
$$

where $\phi_{F}$ is the Eucilidean distance field from the corresponding interface in image $F$ and $\gamma$ is a weight coefficient.

Although Eq. (11) could be solved directly [1], the coupling of the similarity and regularization terms usually makes it computationally complicated. In this work, we solve Eq. (1) based on a Demons framework [11 and rewrite it as

$$
E=\int_{\Omega}\|R-F \circ c\|^{2}+\gamma \int_{\partial \Omega}\left\|\phi_{F} \circ c\right\|^{2}+\frac{1}{\sigma_{x}^{2}} \int_{\Omega}\|c-s\|^{2}+\frac{1}{\sigma_{T}^{2}} \sum_{l=1}^{N} \int_{\Omega} \beta\left\|\nabla s_{l}\right\|^{2},
$$

where the third term is called as the correspondence term and $\sigma_{x}$ accounts for a spatial uncertainty. $c$ is usually expressed in a composition of $s$ and a small updated deformation $u$ : for example, $s \circ(\mathrm{Id}+u)$. By introducing the correspondence term, the coupled optimization can be decoupled into a two-step procedure. It starts from an initial displacement field $s^{0}$. The first step is to solve $u^{t}$ by minimizing the first three terms with $s^{t}$ being given. The second step is to solve $s^{t+1}$ by minimizing the last two terms with $c^{t}=s^{t} \circ\left(\operatorname{Id}+u^{t}\right)$ being given.

\section{Results}

\subsection{2-D Synthetic Experiments}

The performance of the proposed algorithm is first evaluated by 2-D synthetic images. Three synthetic cases are designed with complete or incomplete internal interfaces, denoting fissures, as shown in Fig 2 A line A-A is chosen for each case for comparison between registration-predicted displacements and exact solutions.

The deformation field and intensity patterns are designed to deform "fissures" into designated positions while keep undeformed outside of objects. Fig. 3 shows intensity patterns (gray-scale images with intensity range [0, 255], $128 \times 128$ pixel size, and $1 \times 1 \mathrm{~mm}^{2}$ pixel spacing), deformation fields, and registration results for the three cases above. The reference images are presented on the first three panels in the 1st row, respectively, and they are all registered to the same floating image as shown on the last panel in the 1st row. The warped image, difference image and deformation fields are shown from the 2 nd to 4 th rows, respectively, with each column corresponding to each case in Fig. 2, Comparisons of $x-$ and $y$-components of displacements $(u, v)$ between predicted results and exact solutions along A-A are shown from left to right in Fig. 4 for the three cases, 

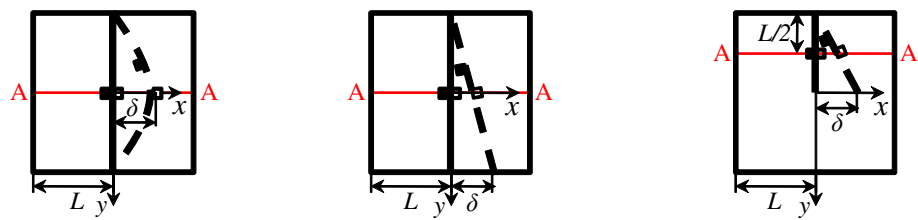

Fig. 2. Sketches for 2-D synthetic cases. Left: Complete Plane-Parabolic, a complete plane "fissure" is deformed into a parabolic surface; Middle: Complete Plane-Plane, a complete plane "fissure" is deformed with changed orientation; Right: Incomplete Plane-Plane, an incomplete plane "fissure" is deformed with changed orientation. The reference line $\mathrm{A}-\mathrm{A}$ is marked in red.

respectively. It can be seen that overall the proposed approach could recover the true deformation field for all the three cases. Discontinuities of both normal $(u)$ and tangent $(v)$ components of displacements for cases with complete or incomplete "fissures" are captured and are consistent with the ground truth.

\section{$3.2 \quad 3-D$ CT Experiments}

In this section, we apply the proposed algorithm to 3-D CT lung datasets with complete lobar fissures. Three pairs of datasets from three human subjects (2 males, 1 female, age: 20-26 years, normal non-smokers) are used. All datasets were acquired with a Siemens Sensation 64 multi-detector row CT scanner (Forchheim, Germany) during breath-holding at $60 \%$ and $80 \%$ of vital capacity under an approved protocol by our institutional review board. Subjects were studied as part of a project seeking to establish a normative lung atlas. We chose these two lung volumes somewhat arbitrarily from datasets with multiple volumes. The volume change (about 0.8 liters) was only slightly greater than a typical tidal volume and thus was suitable for this early evaluation. In-plane dimension is $512 \times 512$ with an approximate spatial resolution of $0.6 \times 0.6 \mathrm{~mm}^{2}$. The z-direction dimension ranges from 600 to 800 with a spatial spacing of $0.5 \mathrm{~mm}$. Complete lobar fissures are detected by using the Pulmonary Workstation 2 (VIDA Diagnostics, Iowa, United States) with the algorithm derived in [12. Similar to 3], we limit our current analysis to the upper and lower lobes of the left lung. Original dataset are cropped to include left lung only. Although we notice that changes in voxel intensity due to respiration might affect registration accuracy [31314], we expect that the influence is small here since volume differences are relatively small. Landmarks located at vessel bifurcations are used to assess registration accuracy. Each registration pair has 40-60 landmarks and they are distributed in the whole left lung. The mismatch errors are $1.25 \pm 1.67$ $(6.49 \pm 5.29), 0.80 \pm 0.39(5.80 \pm 3.71)$, and $1.70 \pm 1.35(8.31 \pm 2.82)$ for three registration pairs, respectively, where units are $\mathrm{mm}$ and numbers in $(\cdot)$ denote errors before registrations. Fig. 5 shows the floating image, reference image, warped image and displacement field from left to right from one subject, representative of the three subjects studied. Discontinuity near the fissure is 

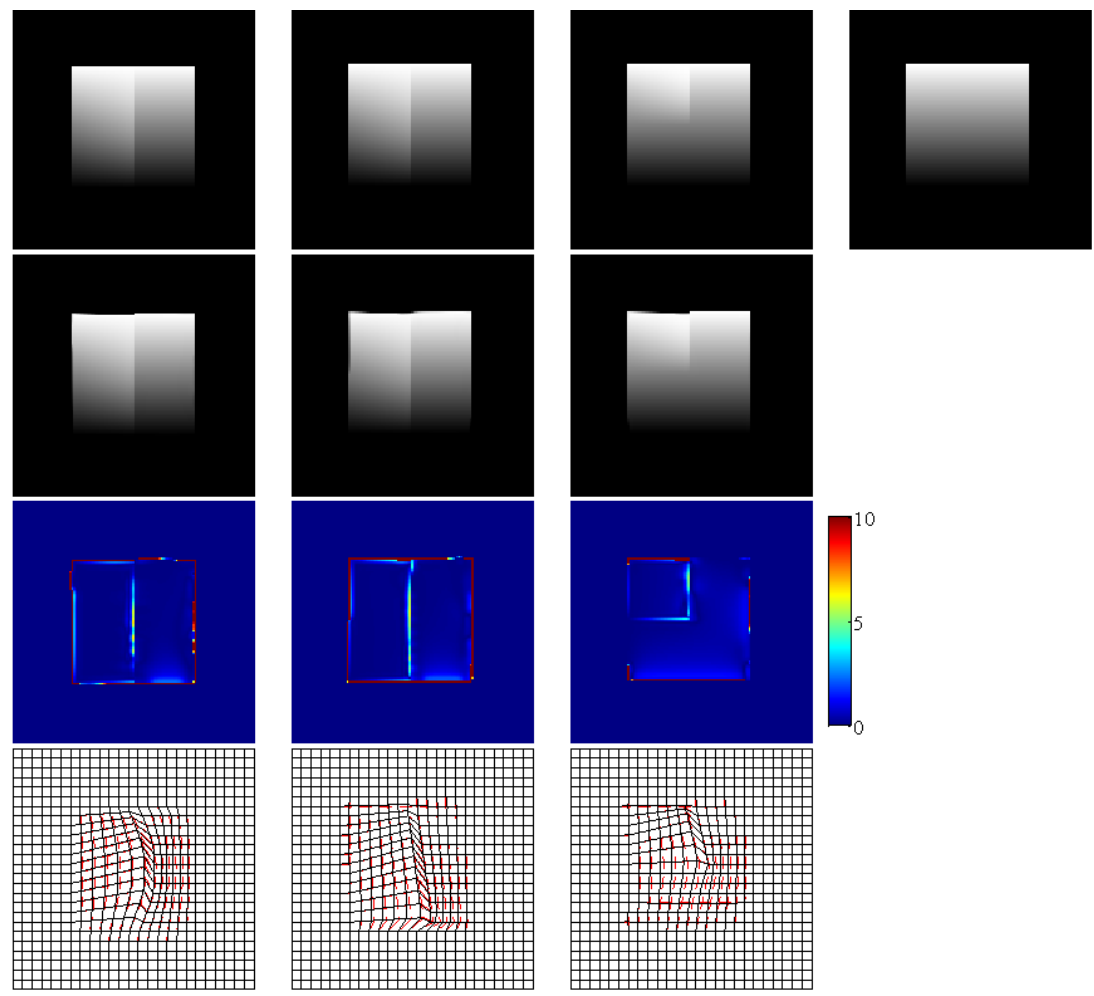

Fig. 3. Results for cases (from 1st to 3rd column): "Complete Plane-Parabolic", "Complete Plane-Plane", "Incomplete Plane-Plane". Reference images are shown and are registered to the same floating image (the last one) in the 1st row. The warped image, difference image, and overlay of exact (red dashed) and predicted (black solid) deformation grids are presented from the 2 nd to 4 th rows.
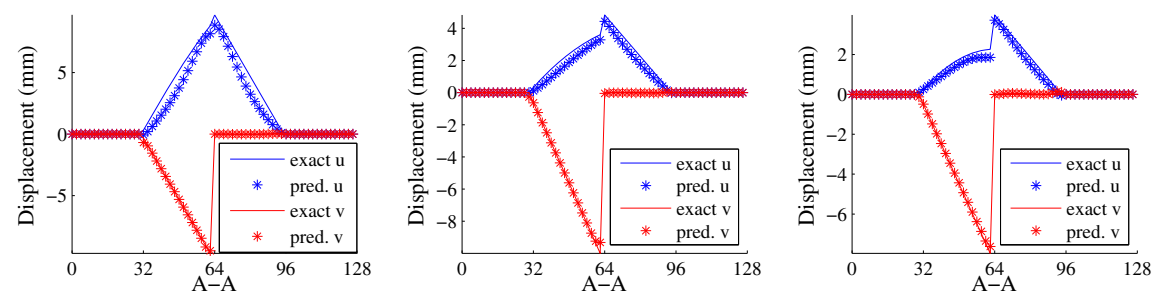

Fig. 4. Comparisons of $u$ and $v$-components of displacements along A-A for three cases: left, "Complete Plane-Parabolic"; middle, "Complete Plane-Plane"; right, "Incomplete Plane-Plane" 

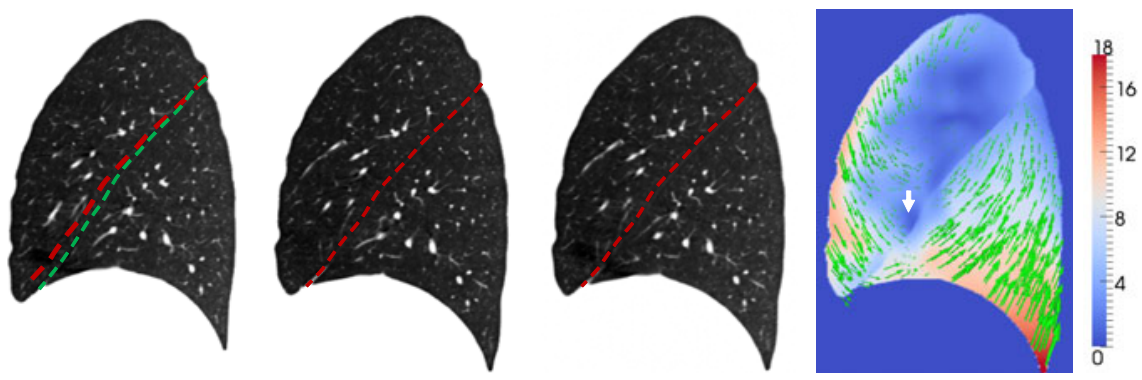

Fig. 5. 3-D lung CT experiment. From left to right: the floating image, reference image, warped image, and displacement field. Oblique fissure is highlighted in green for the floating image and in red for the reference and warped images. The white arrow in the right panel shows a region of low displacement.

clearly visualized. The white arrow in the right panel shows a region with low displacement where motion is restricted by the proximity of the heart.

\section{Discussion and Summary}

In this work, we proposed a novel registration method to capture discontinuities of deformation. We first show that discontinuities can exist in both normal and tangent directions. Such discontinuities are accounted for by a spatially variable diffusive regularization. In addition, we incorporate a distance penalty term into SSD to explicitly match both intensity patterns and interfaces. The new method is evaluated using 2-D synthetic examples with complete or incomplete "fissures", and results show that it is capable of capturing discontinuity of deformation in both normal and tangent directions. The method is then applied to 3-D CT lung datasets with complete fissures. An important next step is to apply the proposed method to datasets with both complete and incomplete fissures to investigate the influence of degree of fissure incompleteness on regional lung mechanics, which will help develop a breathing lung model for computational fluid dynamics simulation of pulmonary air flow [15]16]17.

In the current work, fissure interfaces for both images to be matched are required and a penalty term with the Eucilidean distance is used to match the corresponding interfaces. The interfaces are assumed accurate and investigation of potential effects of their accuracy on registration results would be necessary for future work. An additional issue related to the penalty term is how to prevent gaps or intersections during registrations. Currently we have not checked such uplausible results but we did checked distances of all voxels on the interfaces to ensure that most of them are within one voxel by changing the weight coefficient.

\section{Acknowledgments}

This work was supported in part by NIH Grants R01-HL-064368, R01-EB005823, S10-RR-022421, and R01-HL-094315. 


\section{References}

1. Kabus, S.: Multiple-material variational image registration. $\mathrm{PhD}$ thesis, University of Lübeck (2007)

2. Wu, Z., Rietzel, E., Boldea, V., Sarrut, D., Sharp, G.C.: Evaluation of deformable registration of patient lung $4 \mathrm{DCT}$ with subanatomical region segmentations. Med. Phys. 35(2), 775-781 (2008)

3. Ding, K., Yin, Y., Cao, K., Christensen, G.E., Lin, C.-L., Hoffman, E.A., Reinhardt, J.M.: Evaluation of lobar biomechanics during respiration using image registration. In: Yang, G.-Z., Hawkes, D., Rueckert, D., Noble, A., Taylor, C. (eds.) MICCAI 2009. LNCS, vol. 5761, pp. 739-746. Springer, Heidelberg (2009)

4. Xie, Y., Chao, M., Xing, L.: Tissue feature-based and segmented deformable image registration for improved modeling of shear movement of lungs. Int. J. Radiation Oncology Biol. Phys. 74(4), 1256-1265 (2009)

5. Schmidt-Richberg, A., Ehrhardt, J., Werner, R., Handels, H.: Slipping objects in image registration: improved motion field estimation with direction-dependent regularization. In: Yang, G.-Z., Hawkes, D., Rueckert, D., Noble, A., Taylor, C. (eds.) MICCAI 2009. LNCS, vol. 5761, pp. 755-762. Springer, Heidelberg (2009)

6. Schmidt-Richberg, A., Ehrhardt, J., Werner, R., Handels, H.: Direction-dependent regularization for improved estimation of liver and lung motion in $4 \mathrm{D}$ image data. In: SPIE Medical Imaging, vol. 7623, pp. 76232Y (2010)

7. Rodarte, J.R., Hubmayr, R.D., Stamenovic, D., Walters, B.J.: Regional lung strain in dogs during deflation from total lung capacity. J. Appl. Physiol. 58, 164-172 (1985)

8. Hoffman, E.A., Ritman, E.L.: Effect of body orientation on regional lung expansion in dog and sloth. J. Appl. Physiol. 59(2), 481-491 (1985)

9. Lubner, M.G.: The incomplete fissure sign. Radiology 247(2), 589-590 (2008)

10. Betke, M., Hong, H., Thomas, D., Prince, C., Ko, J.P.: Landmark detection in the chest and registration of lung surfaces with an application to nodule registration. Med. Image Anal. 7, 265-281 (2003)

11. Vercauteren, T., Pennec, X., Perchant, A., Ayache, N.: Diffeomorphic demons: Efficient non-parametric image registration. NeuroImage 45, S61-S72 (2009)

12. Ukil, S., Reinhardt, J.: Anatomy-guided lung lobe segmentation in x-ray ct images. IEEE Trans. Med. Imaging 28(2), 202-214 (2009)

13. Yin, Y., Hoffman, E.A., Lin, C.-L.: Mass preserving nonrigid registration of CT lung images using cubic B-spline. Med. Phys. 36(9), 4213-4222 (2009)

14. Yin, Y., Hoffman, E.A., Lin, C.-L.: Local tissue-weight-based nonrigid registration of lung images with application to regional ventilation. In: SPIE Medical Imaging, vol. 7262 , pp. $72620 \mathrm{C}(2009)$

15. Lin, C.-L., Tawhai, M.H., McLennan, G., Hoffman, E.A.: Characteristics of the turbulent laryngeal jet and its effect onairflow in the human intra-thoracic airways. Respir. Physiol. Neurobiol. 157(2-3), 295-309 (2007)

16. Lin, C.-L., Tawhai, M.H., McLennan, G., Hoffman, E.A.: Multiscale simulation of gas flow in subject-specific models of the human lung. IEEE Eng. Med. Biol. Mag. 28(3), 25-33 (2009)

17. Yin, Y., Choi, J., Hoffman, E.A., Tawhai, M.H., Lin, C.-L.: Simulation of pulmonary air flow with a subject-specific boundary condition. J. Biomech. (2010), doi: $10.1016 /$ j.jbiomech.2010.03.048 